\title{
La Representación Simbólica de la Responsabilidad Social Empresarial (RSE): El Caso Santa Marta
}

\author{
Symbolic representation of corporate social \\ responsibility (csr ): case Santa Marta
}

\section{Devinso Jiménez Sierra}

Filósofo, Investigador y Docente universitario, Maestrante en gerencia educativa. Líder del grupo de investigación RESPONSABILIDAD CUNISTA categorizado en $\mathrm{C}$ por Colciencias. Su línea de investigación es la RSE. Ha sido ponente internacional en distintos eventos académicos. Devinso_jimenez@cun.edu.co
Leonardo Pérez Suescún Contador Público. Administrador de Empresas. Especialista en Finanzas. Especialista en Administración de la Informática Educativa. Magíster en Desarrollo Sostenible y Medio Ambiente. Candidato a Doctor en Ciencias Económicas y Administrativas. Doctorando en Ciencias Gerenciales. Docente de la Corporación Unificada Nacional de Educación Superior. Avenida del Libertador $N^{\circ}$ 29-13. 3008862711.

Leonardo_perez@cun.edu.co

Información del artículo: recibido: Diciembre de 2015, aceptado: febrero de 2016

https://doi.org/10.17081/invinno.4.2.2487 


\title{
Resumen
}

$\mathrm{E}$ 1 artículo analiza la representación simbólica respecto al concepto Palabras clave: de Responsabilidad social empresarial (RSE). El enfoque teórico se realiza desde la perspectiva del signo en C. Peirce y su desarrollo del símbolo, apoyado en la teoría de las funciones del lenguaje de Karl Buhler; función expresiva, función apelativa y función representativa, adherido a la RSE, ética, responsabilidad, proposición de la función argumentativa de K. Popper. El artículo se apoya, representación, además, en la conceptualización de la empresa moderna que hace Adela signo Cortina como una institución económica de incidencia social, en donde se desarrolla una actividad peculiar direccionada desde un horizonte misional formal o no formal con capacidad gerencial y toma de decisiones. Estás asimetrías conceptuales se toman para mostrar el reduccionismo recurrente del concepto de RSE enmarcado solo desde sus dimensiones y no tomado como un proceso holístico que involucre todos los grupos de interés asociados a una organización.

\begin{abstract}
T he article analyzes the symbolic representation regarding the concept Keywords: of corporate social responsibility (CSR). The theoretical approach supported by the theory of language functions of Karl Buhler ; expressive function, appellate function and representative function, adhered to the proposition of argumentative function of K. Popper. The article also supports the conceptualization of the modern enterprise that makes Adela signs Cortina as an economic institution of social impact, where a peculiar activity directed from a formal or missionary horizon with management skills and decision-making develops. Conceptual asymmetries are taken to show the recurring reductionism of the CSR concept framed only from its size and not taken as a holistic process involving all interest groups associated

CSR, ethics, responsibility, symbol, representation,
\end{abstract}




\section{Introducción}

Tr

a ampliación de una visión del mundo más allá del capitalismo ha puesto en discusión la fragilidad del individuo en términos de protección. Ante la necesidad de garantizar la protección de los ciudadanos por encima de los deberes del Estado, ha surgido como discusión el tema de responsabilidad social empresarial, en adelante RSE. Sin embargo, y a pesar de ser un tema en discusión por más de veinte años, el modelo actual de RSE se ha acomodado a las necesidades vitales de las compañías. Las organizaciones le apuestan a un modelo de rentabilidad económica basado en la gestión de sus ingresos haciendo ver a la RSE como un instrumento que refuerza sus deberes estrictamente comerciales sin potencializar el impacto de la RSE como motor del desarrollo sostenible desde la equidad [1].

Para establecer los antecedentes es importante conocer algunas variaciones prácticas del marco conceptual de la RSE, debido a que es posible que las prácticas corporativas atiendan particularmente a unos grupos de interés sin establecer como tal un ejercicio dinámico de la RSE. En la actualidad, nos encontramos inmersos en una sociedad cada vez más globalizada. Uno de los alcances de la globalización es que las consecuencias de nuestras acciones impactan cada vez a una mayor cantidad de personas en una cantidad cada vez más amplia de lugares. Cuando asumimos esta realidad, estamos ante lo que se conoce como Responsabilidad Social [2].

El artículo pretende mostrar, desde la perspectiva del signo en Peirce, los elementos proyectivos de la RSE y su proceso de normatización y convencionalismo que generan su representación simbólica.

\section{Métodos y herramientas}

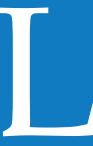

a investigación es de tipo descriptiva. Se estableció una medición de la representación simbólica tomando como referencia la tabla de signos de Pierce (ver Gráfica 3). En ella se cruzan entres sí las variables: representación, fundamento e interpretación. Para esta investigación solo nos concentraremos en las variables asociadas a interpretación y fundamentos puesto que son estas las que permiten deducir un signo de clase: Símbolo [3].

En una primera fase, se realiza una validación de la base de datos de la cámara de comercio de la ciudad de Santa Marta para determinar los criterios de selección de la población objeto de estudio. En este caso se determinan los establecimientos de comercio pequeños, medianos y grandes (ver Tabla 3) con registro mercantil vigente a corte de diciembre de 2014 por medio de un muestreo aleatorio simple (MAS) dentro de un universo de 11.513 establecimientos. Luego se diseña el instrumento de medición, se valida su nivel de confiabilidad, se aplica una prueba piloto y, posteriormente, se aplica el instrumento de medición diseñado como un formulario y aplicado bajo la técnica de encuesta en línea. En la tercera fase se realiza el análisis de los resultados. El instrumento mide una variable de estudio y cuatro dimensiones; con una batería de veintiún indicadores discriminados como lo indica la Tabla 1.

El instrumento fue validado en función de la evidencia de constructo. La validez de constructo "se refiere a qué tan exitosamente un instrumento representa y mide un concepto teórico", y "parte del grado en el que las mediciones del con- 
cepto proporcionadas por el instrumento se relacionan de manera consistente con otras mediciones de otros conceptos, de acuerdo con modelos e hipótesis derivadas teóricamente (que conciernen a los conceptos que se están midiendo)". El proceso de validación de la escala se hizo mediante análisis factorial por análisis de componentes principales, lo que redujo la versión original de seis dimensiones y 26 ítems a solo 4 dimensiones y 21 ítems. Por otro lado, la encuesta ha demostrado una confiabilidad suficiente. La confiabilidad de un instrumento se refiere "al grado en que su aplicación repetida al mismo sujeto $u$ objeto produce resultados iguales". En ese sentido, la escala alcanzó una confiabilidad de 0,812, medida con el coeficiente alfa Cronbach.

Tabla 1. Tabla de variable VARIABLE: Representación simbólica de RSE

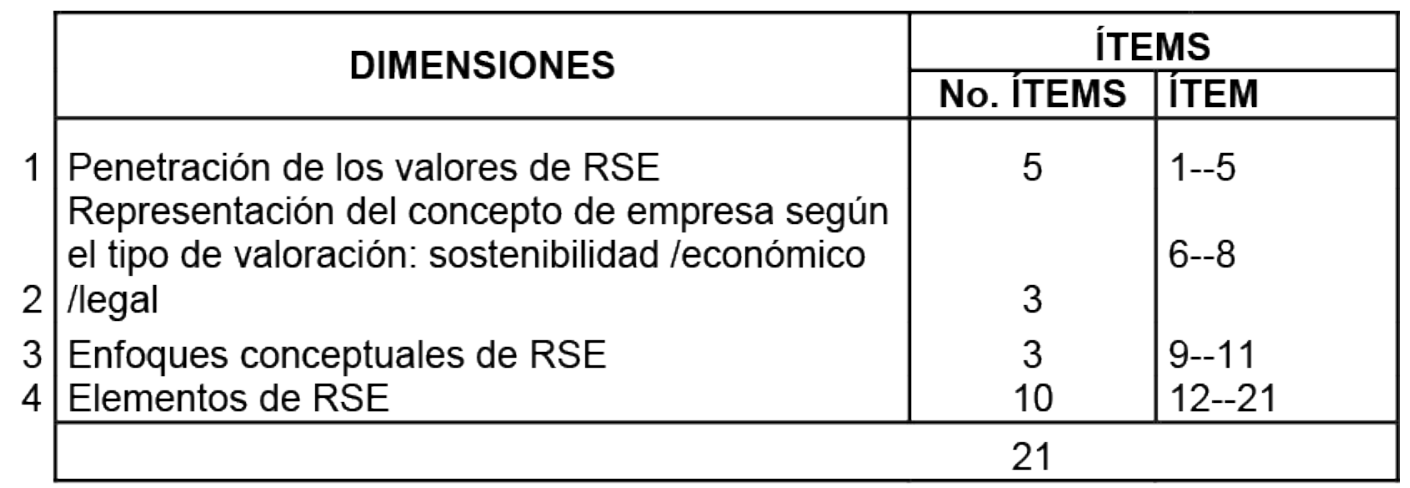


Tabla 2. Fases del proyecto

FASE 1 Análisis sobre la base de datos. Diseño, validez, confiabilidad y aplicación de

FASE 2 instrumento.

FASE 3 Análisis de resultados.

Fuente propia

Tabla 3. Ficha técnica cálculo de muestreo

Universo: 11.513 establecimientos de comercio

Margen de error: $6 \%$

Nivel de confianza: $95 \%$

Nivel de heterogeneidad: $50 \%$

Tamaño de la muestra: 261 establecimientos de comercio.

La estimación se hizo por medio de cálculo electrónico.

Fuente propia

Tabla 4. Empresas en la muestra

\begin{tabular}{lr} 
TAMAÑO DE EMPRESAS & Número de empresas en la muestra \\
\hline Pequeña empresa (6-50 trabajadores) & 32 \\
Mediana (51- 500 trabajadores) & 126 \\
Grande (más de 500 trabajadores) & 103 \\
\hline Fuente propia &
\end{tabular}

\section{Resultados}

Los resultados de la investigación mi-

dieron, en una primera dimensión, los valores de penetración de RSE en referencia a cinco indicadores (ver tabla). Cabe resaltar que el segundo indicador muestra una apropiación del significado de la RSE en un nivel altamente significativo que contrasta con los procesos de certificación afines a RSE, en las que solo ocho empresas, del total de la muestra, realizan procesos de certificación afines. Otro aspecto importante que se decanta en la investigación es la poca identificación de inclusión de aspectos de RSE en la identidad estra- tégica de las empresas. La Gráfica 1 nos muestra los picos en relación a la variación máxima y la poca linealidad de los aspectos medidos en esta dimensión. 
Tabla 5. Matriz de puntuación ponderada de medición

\begin{tabular}{cc} 
PUNTUACIÓN & NIVELES DE MEDICIÓN \\
\hline $3.4-5 \mathrm{~A}$ & LTAMENTE SIGNIFICATIVA \\
$1.7-3.3$ & SIGNIFICATIVA \\
$0.1-1.6$ & POCO SIGNIFICATIVA \\
\hline
\end{tabular}

Fuente propia

Tabla 6. PENETRACIÓN DE VALORES DE RSE

PONDERADO

Recordación de identidad estratégica y el futuro

1 preferido de la empresa.

Apropiación sobre el significado de la

2 responsabilidad social empresarial.

Conocimiento sobre la inclusión de aspectos de

RSE en identidad estratégica y el futuro

3 preferido.

Reconocimiento y exposición pública de compromisos éticos por medio de materiales institucionales, por internet o de otra manera

4 que sea adecuada a sus grupos de interés.

5 Certificaciones ISO 26000 u otras de RSE.

\section{Gráfica 1. PENETRACIÓN DE VALORES DE RSE}

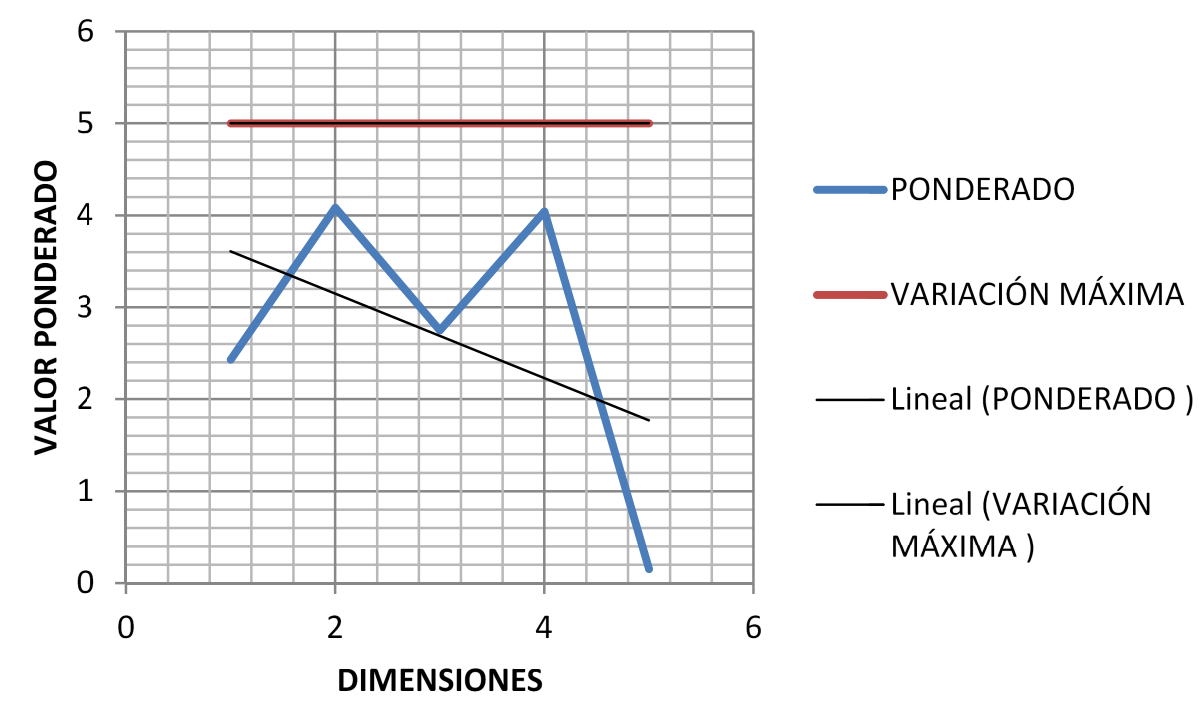

Fuente propia 


\section{Gráfica 2. PENETRACIÓN DE VALORES DE RSE}

En una segunda dimensión, se toman tres conceptos de empresa bajo tres enfoques que valoran lo económico, lo legal y lo sostenible. La definición de cada enfoque corresponde con un tipo de interpretación sobre el concepto de empresa que al cruzarse con un fundamento de la tabla de clase de signos de Pierce (Ver Tabla 7) nos da el tipo de símbolo con el que identifican a la empresa, asignando una ponderación según la matriz de medición que se estableció para esta investigación. De este modo, podemos analizar que el $60 \%$ de las empresas en cuestión sobreponen una valoración legal al concepto

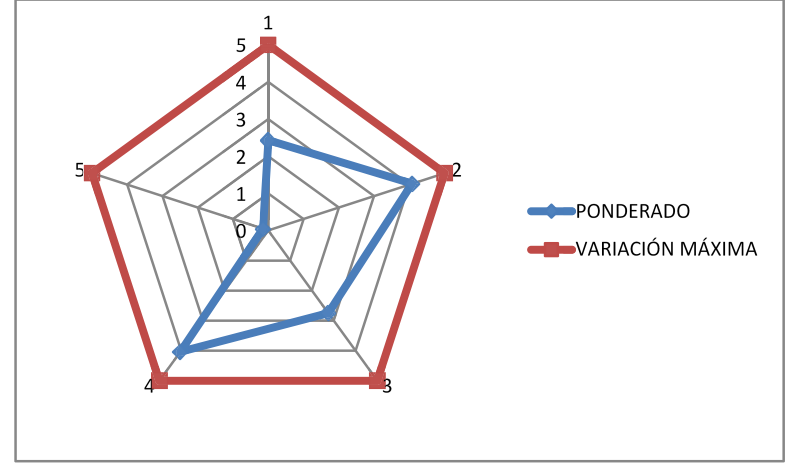

de empresa, es decir, identifican que el concepto de empresa es inherente a la relación legal de la empresa, por lo menos en términos de representación. Le dan una segunda valoración al concepto sostenibilidad con un 35\% y, en términos de representación, valoran el enfoque netamente económico en menor medida, con solo un 3,4\%. Es decir, conceptualizan la empresa: primeramente en términos legales, luego en términos de la sostenibilidad y, por último y en muy poca proporción, en términos de la valoración económica.

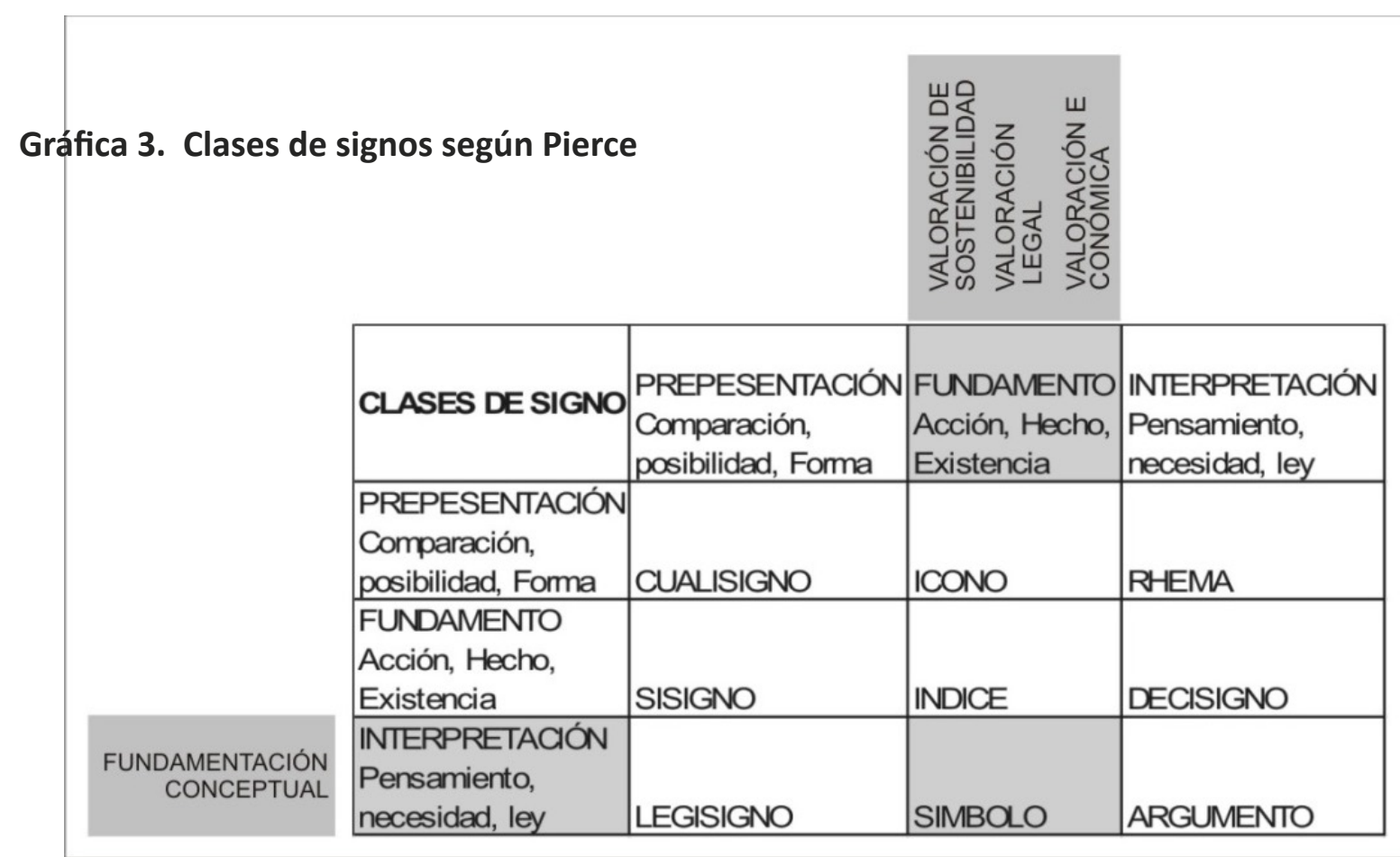

Fuente propia, desde el enfoque de Pierce. 
Tabla 7. Representación del concepto de empresa según el tipo de valoración

\begin{tabular}{|c|c|c|c|c|}
\hline ENFOQUES CONCEPTUALES R & ESULTADO & $\begin{array}{c}\text { TIPO DE } \\
\text { VALORACIÓN }\end{array}$ & PONDERADO & PORCENTAJE \\
\hline $\begin{array}{l}\text { 1. El ejercicio profesional de una actividad } \\
\text { planificada con la finalidad de intermediar en } \\
\text { el mercado de bienes y servicios buscando el } \\
\text { mayor nivel de rentabilidad. }\end{array}$ & 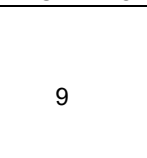 & $\begin{array}{l}\text { VALORACIÓN } \\
\text { ECONÓMICA }\end{array}$ & 0,172413793 & $3,4 \%$ \\
\hline $\begin{array}{l}\text { 2. Una institución económica de incidencia } \\
\text { social, en donde se desarrolla una actividad } \\
\text { peculiar direccionada desde un horizonte } \\
\text { misional bajo principios de sostenibilidad. }\end{array}$ & 93 & $\begin{array}{l}\text { VALORACIÓN DE LA } \\
\text { SOSTENIBILIDAD }\end{array}$ & 1,781609195 & $35,6 \%$ \\
\hline $\begin{array}{l}\text { 3. Un conjunto de elementos y factores } \\
\text { humanos, técnicos y financieros, localizados } \\
\text { en uno o varias unidades físico-espaciales o } \\
\text { centros de gestión ordenados según su } \\
\text { estructura organizativa bajo el más estricto } \\
\text { cumplimiento legal. }\end{array}$ & 159 & VALORACIÖN LEGAL & 3,045977011 & $60,9 \%$ \\
\hline
\end{tabular}

Fuente propia.

\section{Gráfica 4. Valoración del concepto de empresa}

En una tercera dimensión, se cruzan los conceptos de la RSE desde las valoraciones económicas, legales y de sostenibilidad, para determinar el tipo de representación que desde la dirección de la empresa se tiene de la RSE. Es de anotar que al cambiar los enfoques conceptuales también cambiaron las valoraciones de representación, de este modo el $64 \%$ de la muestra considera que su empresa se identifica, en términos de RSE, con una valoración de sostenibilidad, el 24\% considera que su enfoque de RSE es más económico y solo el $10 \%$ considera que su enfoque de RSE es de valoración legal. Son incongruentes los resultados en términos de valoración de la RSE vs. la valoración conceptual de empresa, cuando estas debieran coincidir sucede un alto índice de dispersión.

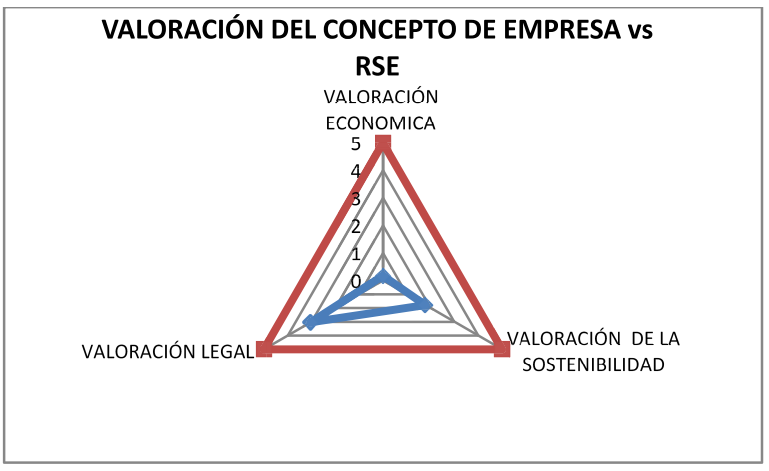


Tabla 8. Enfoques conceptuales de RSE

\begin{tabular}{|c|c|c|c|c|c|}
\hline $\begin{array}{l}\text { ENFOQUES CONCEPTUALES } \\
\text { DE RSE R }\end{array}$ & ESULTADO & $\begin{array}{l}\text { TIPO DE } \\
\text { VALORACIÓN }\end{array}$ & PONDERADO & $\begin{array}{l}\text { VARIACIÓN } \\
\text { MÁXIMA }\end{array}$ & PORCENTAJE \\
\hline $\begin{array}{l}\text { 1. La responsabilidad social de las } \\
\text { empresas es aumentar los } \\
\text { beneficios para sus propietarios o } \\
\text { accionistas conforme a las reglas } \\
\text { básicas de la sociedad en } \\
\text { términos de cumplimiento de la } \\
\text { ley y siguiendo las costumbres } \\
\text { éticas. }\end{array}$ & 64 & $\begin{array}{l}\text { VALORACIÓN } \\
\text { ECONOMICA }\end{array}$ & 1,2 & 5 & $24,50 \%$ \\
\hline $\begin{array}{l}\text { 2. Es la administración de un } \\
\text { negocio de forma que cumpla o } \\
\text { sobrepase las expectativas éticas, } \\
\text { legales, comerciales y públicas } \\
\text { que tiene la sociedad frente a una } \\
\text { empresa. }\end{array}$ & 28 & $\begin{array}{l}\text { VALORACIÓN } \\
\text { LEGAL }\end{array}$ & 0,56 & 5 & $10,70 \%$ \\
\hline $\begin{array}{l}\text { 3. La responsabilidad social } \\
\text { empresarial es el compromiso que } \\
\text { asume una empresa para } \\
\text { contribuir al desarrollo económico } \\
\text { sostenible por medio de la } \\
\text { colaboración con sus empleados, } \\
\text { sus familias, la comunidad local y } \\
\text { la sociedad en pleno, con el } \\
\text { objeto de mejorar la calidad de } \\
\text { vida. }\end{array}$ & 169 & $\begin{array}{c}\text { VALORACIÓN DE } \\
\text { LA } \\
\text { SOSTENIBILIDAD }\end{array}$ & 3,23 & 5 & $64,70 \%$ \\
\hline
\end{tabular}

\section{Gráfica 5. Valoración del concepto de RSE}

En otro momento de la investigación, se tomaron las dimensiones 3 y 4 (representación de la empresa y de la RSE) y se calculó el coeficiente de correlación de Pearson. Este es un coeficiente matemático que oscila entre un valor -1 y un valor 1 . Entre más se acerca la proporción a 1 más positiva es la correlación, entre más se acerca al -1 más negativa es la correlación. Analizando los resultados del coeficiente, nos damos cuenta de que la correlación es negativa, al

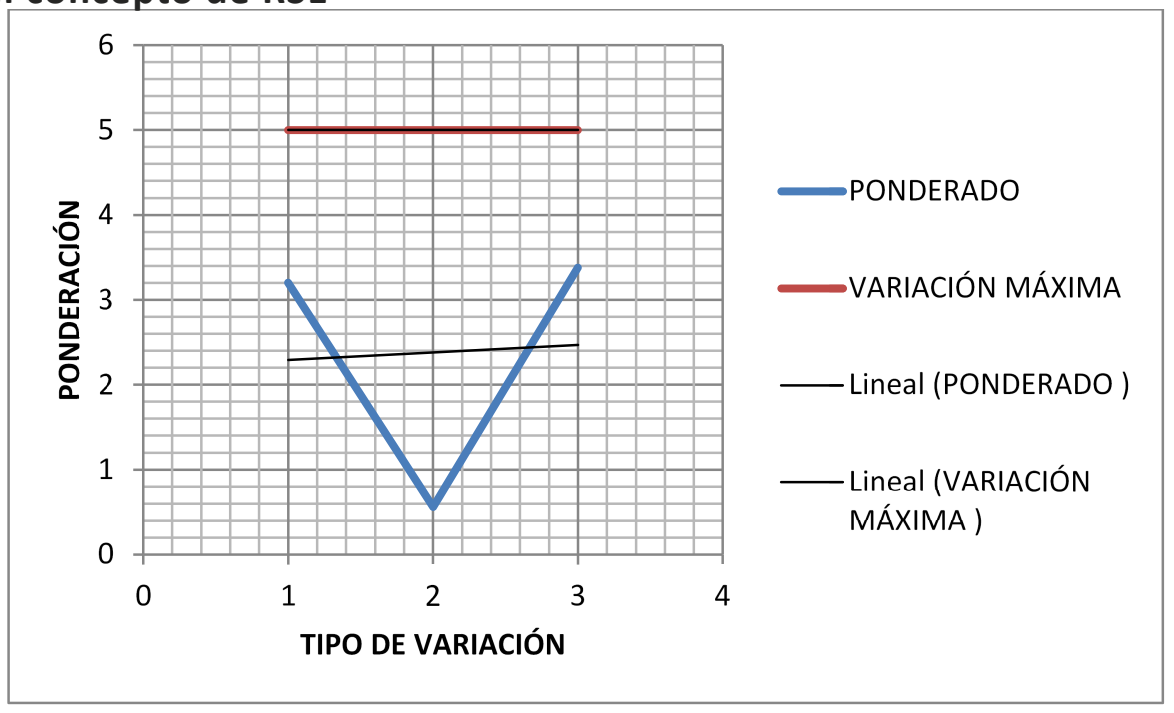

Fuente propia

ser -0.01606 (ver Tabla 9), lo que indica que las valoraciones del concepto de empresa difieren de la valoración del concepto de RSE de la empresa, lo que no es muy coherente en términos de adaptación de políticas de RSE, puesto que se da una no correlación entre las mismas. 
Tabla 9. Enfoque de empresa vs. Enfoque RSE

\begin{tabular}{ccc} 
& $\begin{array}{c}\text { ENFOQUES CONCEPTO DE } \\
\text { EMPRESA }\end{array}$ & $\begin{array}{c}\text { ENFOQUES CONCEPTUALES } \\
\text { DE RSE }\end{array}$ \\
\hline & $\mathbf{X}$ & $\mathbf{Y}$ \\
VALORACIÓN ECONÓMICA & 0,171 &, 2 \\
VALORACIÓN DE LA \\
SOSTENIBILIDAD \\
VALORACIÓN LEGAL & 1,783 &, 56 \\
\hline COEFICIENTE DE CORRELACIÓN DE \\
PEARSON
\end{tabular}

En una cuarta dimensión, se midió la valoración que tienen las empresas sobre diez aspectos relacionados con la responsabilidad social empresarial. En este caso se les pidió que en una escala de 1 a 10 nos dieran el valor que tenía cada aspecto en su empresa. En los resultados volvemos a encontrar que el factor económico es el que presenta mayor valoración para la empresa con un 9,1 sobre 10 , lo sigue otro aspecto altamente relacionado con el primero: la maximización de utilidades. Hay que resaltar que todas las valoraciones, excepto las prácticas filantrópicas, están por encima del 6,8 sobre 10 , lo que muestra un interés medio por la mayoría de los aspectos producto de la medición.

Tabla 10. Elementos de RSE según el nivel de importancia de las organizaciones

\begin{tabular}{llc}
\hline & ESCALA 1-10 \\
1 Factores económicos de la empresa & 9,1 \\
2 Maximización de utilidades & 9 \\
3 Respuestas a quejas de clientes & 8,5 \\
4 Condición legal de los proveedores & 7,9 \\
5 & Relacionamiento con la competencia & 7,7 \\
6 Bienestar laboral & 7,4 \\
7 Relacionamiento con el sector público 7 &, 3 \\
8 Aspectos ambientales & 7,1 \\
Relacionamiento con el entorno & 6,9 \\
10 comunitario & 4,23 \\
\hline
\end{tabular}

Fuente propia 
Gráfica 6. Valoración de elementos de RSE

\section{VALORACIÓN DE ELEMENTOS DE RSE}

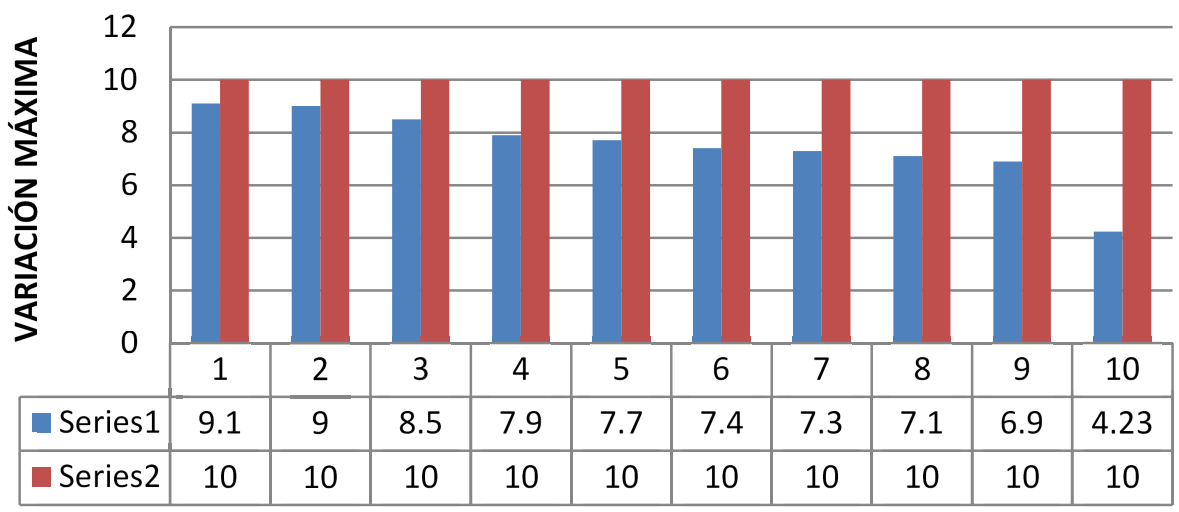

Fuente propia

Gráfica 7. Valoración de elementos de RSE

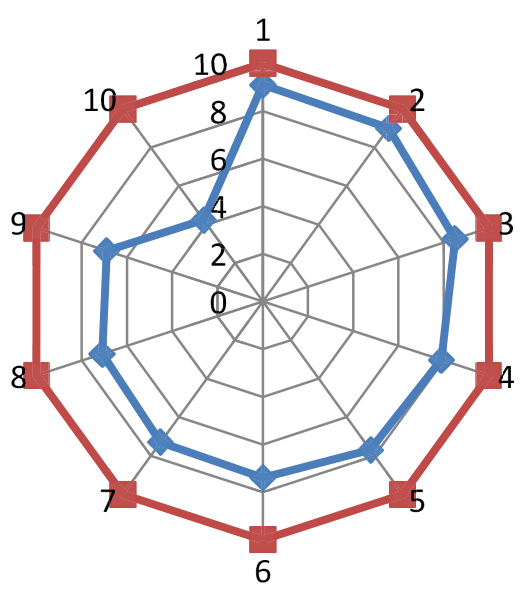

Fuente propia

\section{Discución} Perspectiva de la RSE

En su forma básica, la RSE se refiere a la idea de que, en particular, las grandes organizaciones del sector empresarial, debido a su mayor potencia y efectos en las sociedades de todo el mundo [4], tienen o deberían disponer de un conjunto más amplio de responsabilidades hacia la sociedad en general, que van más allá del mero interés económico en términos legales y en general a las obligaciones de la empresa [5]. Aunque la demanda de RSE tradicionalmente se ha referido a las grandes empresas, el concepto de RSE se aplica a todas las empresas y organizaciones, y se ha convertido en un reto importante para cualquier empresa independientemente de su tamaño [4].

Tradicionalmente, la empresa fue considerada socialmente responsable de cumplir con las expectativas de la sociedad, contribuyendo a la economía 
en general y a la creación de riqueza para la sociedad. Al principio, la función y responsabilidad de la empresa fue producir bienes y servicios, proporcionar puestos de trabajo y ser rentable por cualquier medio posible, independientemente de las consecuencias humanas y ambientales, siempre y cuando estuviese dentro del ámbito de la ley [6].

En concordancia con esta visión básica de la empresa y sus responsabilidades, el muy citado economista estadounidense [7] argumenta que la única responsabilidad social de las empresas es aumentar los beneficios para sus propietarios o accionistas, esto conforme a las reglas básicas de la sociedad en términos de cumplimiento de la ley y siguiendo las costumbres éticas actuales. Según Friedman, las actividades sociales en términos de donación de dinero a buenas causas y el hacer frente a otros problemas sociales son consideradas una pérdida de "dinero y una distracción del propósito fundamental de una sociedad".

Este punto de vista clásico de la empresa y sus responsabilidades es ampliamente citado como un claro contraste con las tendencias contemporáneas de la RSE. Sin embargo, todavía desempeñan un papel en la definición de RSE, ya que forma la base de conceptualizaciones contemporáneas y de la comprensión de la RSE.

La literatura contemporánea sobre la RSE considera a las empresas responsables de un amplio grupo de intereses más allá de los propietarios o accionistas de la empresa. Esta concepción se basa en la teoría de la gestión de intereses que define al interesado como cualquier grupo o individuo que puede afectar o es afectado por el logro de los objetivos de la empresa con el fin de obtener ganancias y mantenerse competitivos con altos estándares de calidad. Las empresas deben identificar y actuar de acuerdo con las demandas y expectativas de sus grupos de interés (consumidores, empleados, inversores, gobiernos, proveedores, miembros de las comunidades locales, ONG, etc.) [4]. En este sentido, la responsabilidad de una empresa se define por sus grupos de interés.

De acuerdo con la visión más amplia de la RSE, las empresas son una parte integral de la sociedad en la que operan. Sus acciones afectan a la sociedad en general y por lo tanto son consideradas responsables de su impacto en la sociedad en su conjunto. Las empresas deben servir de manera constructiva a las necesidades de la sociedad, a satisfacción de la sociedad. A fin de obtener certificaciones internacionales, como la norma ISO 26001 de responsabilidad social, que amplíen sus procesos de participación en mercados altamente exigentes, al hablar de las responsabilidades de las empresas para con la sociedad, se incluye a los grupos de interés en su sentido original (propietarios $\mathrm{o}$ accionistas), pero también se amplía la perspectiva para incluir otros elementos como el medio ambiente, la riqueza general y la calidad de vida en las comunidades insertadas en el contexto geográfico en el que operan las empresas.

En cuanto a la definición internacional de la RSE, la Unión Europea la define como: "Un concepto mediante el cual las empresas integran las preocupaciones sociales $y$ medioambientales en sus negocios, operaciones $\mathrm{y}$ 
en sus interacciones con sus grupos de interés de manera voluntaria" [8]. La Unión Europea especifica las preocupaciones sociales y ambientales como las principales áreas de responsabilidad de las empresas, a hacer frente de forma voluntaria, después de su finalidad económica. RSE se refiere a la decisión voluntaria de las empresas de contribuir en la construcción de una sociedad mejor y un medioambiente más limpio [8], por medio de la gestión, medición e impactos en la sociedad dentro de los ámbitos económico, social y ambiental. Un elemento importante que no puede pasar desapercibido es que la RSE, en un sentido internacional, se basa en un diálogo con las partes interesadas que, entre otras cosas, podría incluir alianzas con diversas instituciones tales como sindicatos, autoridades públicas, fundaciones, centros de pensamiento, ONG y representantes de negocios y organizaciones. El concepto diálogo dentro de la perspectiva de una empresa omnipotente genera un alto nivel de ambigüedad, pero en realidad la RSE lo que busca es establecer una relación cercana de sostenibilidad y eficiencia en respuesta al desgastado modelo de consumo continuo sin techo ni recursos necesarios para sostenerse.

\section{La representación según Peirce}

En términos de referencias, Peirce [3] ha propuesto una clasificación del signo según sus referentes: icono, índice y símbolo. Al primero lo define como un signo que hace referencia a su objeto en virtud de una semejanza o ciertas propiedades de correspondencia con las propiedades del sujeto. El índice es un signo que tiene conexión física con el objeto, por ejemplo, el humo es indicio de fuego, una brújula indica siempre hacia el norte. El símbolo, por último, es un signo arbitrario cuya relación con el objeto se determina por reglas o convenciones. Entre estos me interesa hacer hincapié en el concepto de Símbolo. Peirce [3] también lo definía como "un vehículo que transporta dentro de la mente algo desde afuera. Aquello por lo que está se llama su objeto; aquello que transporta, su significado; y la idea a la que da lugar, su interpretante."

La fundamentación de un signo que denota representación legal, en términos de Peirce: legisigno, conduce hacia un símbolo. Este requiere de la condición de legitimidad, que puede tomarse como convención. Para que un signo pueda representar debe haber un acuerdo, llámese político, cultural, científico, religioso, que prescriba su condición de representación frente a quien lo interpreta. El canal de interpretación de un signo/legisigno es la función argumentativa.

En el escrito On the Algebra of Logic, Peirce afirma que el signo está ligado al objeto en virtud de una asociación mental y depende por tanto de un hábito. Estos signos son siempre generales $\mathrm{y}$, con frecuencia, convencionales y arbitrarios. Al pensar en la representación simbólica de la responsabilidad social empresarial podemos denotar factores de representación asociadas a un índice: la ética desde la perspectiva axiológica. Pero antes de incluir los aspectos formales de la RSE es importante describir las funciones básicas del lenguaje para identificar las que competen al proceso de representación simbólica.

En el plano de las funcio- 
nes básicas del lenguaje tenemos los postulados de Karl Buhler [9], precursor del funcionalismo lingüístico alemán. Este discriminó tres tipos de funciones, a saber: la función expresiva, la función apelativa y la función representativa. Las dos primeras actuantes en la vida animal en general, la tercera es de uso exclusivo del ser humano, puesto que posibilita la construcción mejorada y compleja de cada aspecto que se representa. Anteriormente, Karl Popper, en una publicación de 1977, había fundamentado que la función argumentativa era un elemento esencial en el lenguaje codificado, aspecto que se complementaría con la teoría de Buhler creando cohesión teórica.

La función argumentativa genera en la representación el móvil para la fijación de convicciones, establecer procesos de aceptación de postulados constrictivos capaces de construir una estructura conceptual que da legalidad a lo representado. El principio de la ciencia es el de asumir los postulados generales prescritos por el cumplimiento de una ley expresada en argumentos constrictivos a los que llamamos verdad científica.
Representación simbólica de la RSE

El símbolo es un signo de clase legisigno, como lo he afirmado, que necesita una adecuación según convenciones para su aceptación. El móvil de su convicción es la función argumentativa. Cuando se habla de la responsabilidad social empresarial se prescribe como un concepto de naturaleza figurativa asociado a distintas dimensiones a las que denominaré índices, en particular: 1) dimensión económica, 2) dimensión de sostenibilidad y 3 ) dimensión social. El modo de asociación simbólica es la función argumentativa, entre ellos asumiré los postulados que describen la RSE como mecanismo de cohesión social $[4,5]$.

La sociedad moderna ha construido un discurso normativo en favor de la armonización de los valores éticos civiles en el ámbito de la empresa a la que hemos denominado RSE, esta proyección no permite una apropiación vinculante y determinante de los temas asociados a la RSE. A tal punto que nos hemos quedado con una representación simbólica de la RSE bajo conceptos generalizados enmarcados dentro del establecimiento de dimensiones primarias (económico/de sosteni- bilidad/social) creando un legisigno normativo convenido en los distintos tipos de certificaciones internacionales (SA8000, iso26000, Global Reporting Initiative (GRI), Pacto Global de Naciones Unidas, AA1000, Lineamientos para Empresas Multinacionales OCDE, Principios Globales de Sullivan, entre otros) y soportado en las diferentes corrientes teóricas en torno a la RSE. Estos procesos normativos son tomados como modelos voluntarios de asunción de la RSE, existen, se difunden, se argumentan (función argumentativa), 37 pero no hay coerción de aplicación, lo que genera una representación simbólica desde sus dimensiones primarias mas no desde todos los grupos de interés que la gravitan, mermando los niveles de impacto de la RSE.

Todo símbolo tiene índices, en el caso de la representación que nos hacemos de la RSE son lo económico/ambiental/ social. Estos conceptos actúan como proyección de los contenidos de la RSE. Al proyectar los conceptos del legisigno vía función argumentativa nos quedamos con microelementos de la RSE, mas no con la totalidad de factores fun- 
dantes de la RSE. Este nales no permite incorpocomplejo fenómeno de rar en la representación representación simbólica de la misma los demás generaliza los conceptos elementos dinámicos asociados a él sin que como son la relación entre permita una discusión a dimensiones y la incorpoprofundidad. ración de más grupos de interés (stakeholders) en

Referenciar la RSE solo a la visión holística de la lineamientos dimensio- empresa moderna.

\section{Coclusión}

L

a empresa moderna asume un reto histórico en la construcción de sentido de los procesos de cohesión social, para ello el concepto de RSE se erige como un instrumento de tránsito que nos acerca a la construcción de una sociedad con más equidad y justicia. Adela Cortina [10] ha introducido en la discusión de la RSE la concepción ética bajo la mirada de la modernidad sin restringir el uso de la libertad, pero asumiendo valores replicables desde la responsabilidad civil y su proyección en el sector empresarial.

Pero esta visión moderna de la RSE en muchos casos se estanca en proyecciones conceptuales dominadas por su alto contenido argumentativo (función argumentativa según Popper). El discurso crea una representación simbólica desde las dimensiones económica/ambiental/ social de primer orden que no permite permear discusiones de fondo que, aunque se encuentran asociadas a dichas dimensiones, no establecen relación directa con los distintos grupos de interés (stakeholders). Esta representación simbólica, como la he llamado, no permite ampliar el margen de conocimiento y por ende de aplicación de una RSE imbricada en generar procesos de cohesión social. Queda abierta la discusión para reconfigurar una nueva representación de la RSE holística e integradora.

\section{Referencias Bibliográficas}

1. A. B. Gómez. Análisis organizacional basado en los lineamientos de la Responsabilidad Social Empresarial. Bogotá: pontificia Universidad Javeriana, 2009.

2. M. P. Valdivia. Análisis Crítico de los Sistemas de Medición de Responsabilidad Social Empresarial. Santiago de chile: Uchile, 2007.

3. C. Pierce, El hombre un signo. Barcelona: España. Crítica, 1988.

4. P. Neergaard, Responsabilidad Social Corporativa. Alemania: Editores Académica. Traducción de Sergio Moral, 2006.

5. A. Carroll, "La Pirámide de la Responsabilidad Social Empresarial: Hacia la Moral en la Gestión de los stakeholders de la organización". Horizonte, pp 39-48, 1991.

6. O. Idowu, \& Filho. Prácticas Globales de la Responsabilidad Social Empresarial. Madrid: Heidelberg, 2009. 
7. M. Friedman, La responsabilidad social de las empresas es aumentar sus beneficios. Detroit: Teorías y Conceptos, 1970 .

8. Comisión Europea. Libro Verde: Fomentar un marco europeo para la responsabilidad social. Comisión de las Comunidades Europeas, 2001. [en línea] consultado octubre 15 de 2012. Disponible en: http://eur-lex.europa. eu/LexUriServ / site/en/com/2001/ com2001_0366en01.pdf

9. K. Buhler. Teoría del lenguaje. Madrid: España. Alianza, 1979.

10. A. Cortina. Ética de la empresa. 5edición. Madrid: España. Trotta, 2000.

Bibliografía de consulta

1. Aristóteles, Acerca del alma. Madrid: España. Gredos, 1987.

2. Aristóteles, Ética a Nicómaco. Madrid: España. Alianza, 2004.

3. H. Cassirer, La ciencia de la cultura. México: México. FCE fondo de cultura económica. Edición conmemorativa 70 aniversario, 2005.

4. M. D. González. La responsabilidad social corporativa o responsabilidad social de la empresa. bilbao: Eko urriaren, 2005.

5. D. Held y A. McGrew. Globalización/Antiglobalización. Ed. Paidós, 2003.

6. E. Kant. Crítica de la razón práctica. Madrid: Alianza, 2000.

7. G. Núñez. La responsabilidad social corporativa en un marco de desarro1lo sostenible. CEPAL, Serie Medio Ambiente y Desarrollo, 2003.

8. K. Popper. Búsqueda sin términos. Madrid: Tecnos.

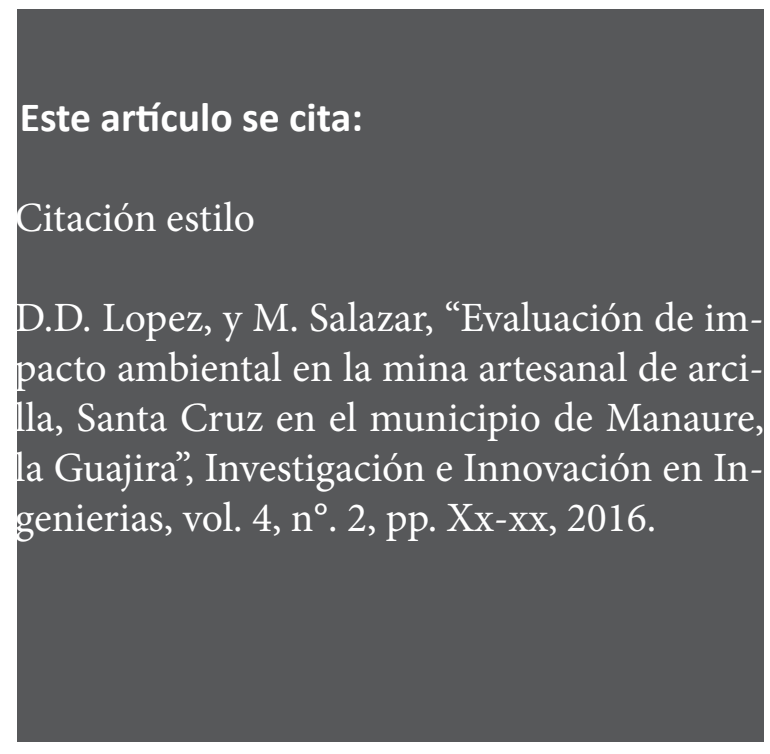

